\title{
La radio comunitaria en el sur de Chile: análisis del discurso de sus actores
}

\section{Community radio in southern Chile: discourse analysis of its actors}

\author{
Juan Domingo Ramírez \\ Docente e investigador del Instituto de Comunicación Social, Facultad de Filosofía y Humanidades, Universidad \\ Austral de Chile. \\ juandomingoramirezc@gmail.com
}

\begin{abstract}
Resumen El artículo aborda conceptos de Comunidad, Asociatividad, Legislación y Uso de Redes Sociales en las radios comunitarias a partir de entrevistas semiestructuradas. Utiliza la Teoría Fundamentada de Glaser y Strauss (1967) y el software Atlas.ti como herramienta de análisis. Plantea una discusión entre lo comunitario y lo ciudadano y la necesidad de observar los movimientos radiales como un proceso. En cuanto a resultados, observa que hay profundidad del discurso de Necesidades y Participación; sin embargo, pocas personas participan en la gestión de las radios. En Asociatividad, participan en organizaciones, pero hay poca conversación con otras emisoras. El Uso de Redes Sociales es masivo (Facebook y Twitter), pero no se relaciona con el crecimiento de estas mismas, en función de aumentar audiencias o multiplicación del mensaje comunitario.
\end{abstract}

\begin{abstract}
Abstrac The article discusses concepts of Community, Associativity, Legislation and Use of Social Networks in Community Radio from semistructured interviews. Use the Grounded Theory of Glaser and Strauss and Atlas.ti software analysis tool. Raises a discussion between the community and citizenship concepts and the need to observe the radial movements as a process. As a result, notes that there depth of speech about Needs and Participation; however, few people participate in management. In Associativity, participating in organizations, but there is little conversation with other stations, which defined as lack. The Use of Social Networks is massive (Facebook or Twitter), but not related to the growth of the same, in terms of increasing audiences or multiplication of Community message.
\end{abstract}

$\begin{aligned} \begin{array}{r}\text { Palabras } \\ \text { Clave }\end{array} & \text { Radios Comunitarias, Comunitarias sur Chile, Teoría Fundamentada, Radios ciudadanas, análisis Atlas.ti. } \\ \text { Key } & \text { Community radio, Community southern Chile, Grounded Theory, CitizenRadio, analysis Atlas.ti. } \\ \text { Words } & \end{aligned}$

○ Agradecimientos: Este proyecto fue financiado por la Dirección de Investigación y Desarrollo, DID, de la Universidad Austral de Chile. S-203-13: Radios comunitarias en Chile: la paradoja de su propiedad y su re-construcción en la era digital. 


\section{La radio comunitaria en el sur de Chile: análisis del discurso de sus actores}

La radio comunitaria es un hacer constante que se relaciona con las personas, su vida, su historia, sus sueños, esperanzas y su expresión. Ignacio López Vigil (2005) ha graficado en metáfora lo que es una radio comunitaria, al señalar que las define por su defensa de la ciudadanía, por su programación o por su capacidad de resolver problemas de la comunidad, entre otras variables.

La visibilización de los movimientos sociales es para Rovira-Sancho (2013) una de las características de estos medios; y tomando la definición de Rowling (2010) usa la denominación de "nanomedios", es decir, medios a pequeña escala, con baja cobertura y casi inexistente presupuesto. Dadas estas características, propone que estos medios no se podrán ver desde teorías rígidas ya que su visibilidad está mucho más radicada en los procesos.

En la actualidad de la radiodifusión no solo se dificulta la definición y el accionar de los medios comunitarios, sino que de la misma radio. ¿Señales de música compartida on line como Spotyfy, LastFm, SkyFm son radios o debiéramos denominarlos solamente "señales"? Las nuevas tecnologías obligan a buscar entonces, dentro de los procesos, las características relevantes de los medios comunitarios, más que en sus acepciones tecnológicas o teóricas.

Esto es, de acuerdo con Cabalín(2010), observar que estos medios en su nacimiento, desarrollo y proyecciones se establecen en espacios pequeños, no masivos, asignándoles un rol en el desarrollo de las comunidades en donde se insertan. Pero Cabalín (2010), Ramírez (2014) y Chaparro (2002) colocan algunos puntos suspensivos acerca de la participación, no por su valor, sino por el contexto de baja asociatividad del conjunto de la sociedad.

Yañez, Collet, Weibel (2011) destacan que los procesos exitosos sí impulsan la asociatividad. Al ser un medio participativo, que valida y comunica distintos niveles de información, señalan que la acción de la radio busca fortalecer el tejido social y la asociatividad comunitaria. En este sentido, tanto radialistas como audiencias tienen interés por hacer eficaces los canales de comunicación y participación. Estos canales articulados en la relación radialistas/vecinos son de tipo participativo vinculante. De este modo, la radio comunitaria funciona como catalizador de los vínculos sociales al interior del territorio local.

Sin embargo, lo comunitario no se da solamente porque una comunidad opere una radio, sino que se dará de acuerdo a los proyectos que generen estos medios y su vinculación con las comunidades. $Y$ eso es tremendamente importante. 
Ramírez (2014) determina que las radios comunitarias en Chile, si bien están en contacto con sus públicos en términos de reunirse con Agrupaciones Culturales, Juntas de Vecinos, Centros de Adultos Mayores y Grupos de Jóvenes, limitan ese contacto a dar espacios para programas, entrevistas o difundir noticias de actividades. No obstante, son muy pocas las emisoras que tienen una participación directa de las audiencias en la gestión de las emisoras.

Surge también la necesidad de generar una reflexión para encontrar nuevas definiciones, no por ésta en sí misma, sino por la necesidad de entender cuáles son las variables que están tras las radios comunitarias, con el fin de dar sustento teórico a los procesos.

Van Oyen, refiriéndose a la participación que tuvieron y tienen las emisoras mineras bolivianas en los procesos sociales de cambio, destaca su importante papel:

\footnotetext{
El proyecto Político Comunicacional de las radios populares y comunitarias no termina en sí mismo, sino que está enmarcado dentro de un proyecto sociopolítico más amplio. La historia de las radios populares enseña que todas nacieron como parte de proyectos de desarrollo, de educación, de alfabetización, de evangelización, de comunicación cultural o cambio sociopolítico. Este proyecto más grande, en función del cual existen las radios populares y comunitarias, lo describen muchas de ellas en términos de a) una nueva democracia: una sociedad más humana basada en la convivencia democrática, lo que implica democratizar el Estado y democratizar la misma sociedad: b) un nuevo desarrollo equitativo e integral, claramente diferenciado de la propuesta neoliberal. (Van Oeyen, 2003)
}

Por lo tanto, esta función se ejerce desde distintas emisoras y proyectos. Vale tanto la radio que evangeliza, la que educa, la que refleja la comunidad, la ciudadanía o la musical. Lo importantes es el quehacer individual y en conjunto de los proyectos, que lleva implícito el proyecto popular de las radios.

\section{Lo comunitario en la sociedad actual}

Las comunitarias se han definido como populares, ilegales, piratas, libres, y en los últimos años se ha instalado el concepto de radios ciudadanas. Este giro a ciudadanas tal vez esté influido por la tecnología y la capacidad de tener una radio por el aire, a la que se le asigna una señal en un entorno geográfico limitado, pero que se transforma en un espacio ilimitado a través de internet. 
Este concepto de radios ciudadanas es aún muy ambicioso, claro está que nos referimos a los términos teóricos del término, ya que en la práctica está todo unido. Por ejemplo, cuando algunos actores hablan de radios ciudadanas, están hablando de una radio que sale de los espacios geográficos de una comuna, un pueblo, que es capaz de conectarse con otras emisoras, generar cadenas, programas compartidos.

En cambio, lo que habíamos denominado antes como comunitaria trataba de una emisora situada dentro de un espacio geográfico, generalmente delimitado por condicionantes técnicos legales, dentro de una comuna o localidad. Esta definición no es valórica, de una radio buena u otra mala, correcta o incorrecta, sino que se trata de datos del discurso y de los usos de términos.

Entonces nos encontramos con un conjunto de emisoras que en términos de Balibar (2013), serán emisoras insurreccionales, es decir, que están generando una ciudadanía activa en defensa del cambio, de un Derecho Humano como lo es el derecho a la comunicación, entendido en términos de Pasquali (2014), como la potestad irrenunciable de no sólo hablar por los medios sino el derecho a fundarlos y gestarlos.

Se podría pensar que todas las radios comunitarias son insurreccionalmente ciudadanas, pero este concepto podría aplicarse -por ejemplo- de mejor manera a algunas emisoras en centros urbanos, con un buen acceso a internet, con un soporte de acción y de teoría en su proyecto; mientras, otras más pequeñas, en comunidades chicas, rurales, étnicas, podrían caber más bien en la clásica emisora eminentemente comunitarias -en el sentido básico- y ejercer una labor más cercana a la comunidad, casi definidas por términos geográficos. Ahora bien, está claro que cada experiencia radial comunitaria tiene mucho de única y está inserta en los cambios sociales, políticos y culturales que experimenta nuestra sociedad.

Bauman advierte que estas nociones de ciudadanía y ciudadano "sientan las bases, como mínimo, para la tolerancia mutua, pero de ningún modo llega tan lejos como para sentar las bases de la mutua solidaridad" (Bauman, 2013, p.37). Es decir, mirado desde los proyectos comunitarios, esta "ciudadanía" deberá ser activa ya que el solo hecho de ocupar un espacio no es garantía de nada. Las identidades/ciudadanías modernas -al decir de Martín Barbero - se van construyendo en la negociación de reconocimiento "desde el nuevo valor de la diferencia que articula la universalidad humana de los derechos, a la particularidad de los muy diversos modos de su percepción y de expresión" (Martín-Barbero, 2007, p. 85). Abrutin señala que incluso estos nuevos conceptos de ciudadanía obligan a abandonar la 
dicotomía nación-ciudadano, "para empezar a pensar en un modelo de ciudadanía más abierto e inclusivo, fundado en la acción más que en la identidad”. (Abrutin, 2006, p. 13). Esto es, al decir de Araya, ir estableciendo el cruce entre cultura y ciudadanía "pues no se trata sólo de la posibilidad de que cada ciudadano tenga que participar en la decisión de los asuntos públicos, sino de la posibilidad de que mi identidad no solo parezca sino que aparezca en lo público" (Araya, 2007, p. 48-49).

Enlazando la ciudadanía con la sociedad actual, Bauman (2010) nos lleva a algunas reflexiones acerca del tipo de modernidad -y por ende, el tipo de ciudadanía- en que están insertos los proyectos comunitarios. Al establecer el concepto metafórico de modernidad líquida, mira los procesos sociales de cambios como una disolución de la "solidez" de los soportes de una sociedad, para cambiarlos por otros. El problema está en el estado actual de la sociedad:

Los sólidos que han sido sometidos a la disolución, y que se están derritiendo en este momento, el momento de la modernidad fluida, son los vínculos entre las elecciones individuales y los proyectos y las acciones colectivos -las estructuras de comunicación y coordinación entre las políticas de vida individuales y las acciones políticas colectivas-. (Bauman, 2010, p. 11-12)

Así, siguiendo este razonamiento, encontramos que esa liquidez va a significar que el individuo - pensado como sujeto de derecho- va a ser más que lo colectivo, individuo más que ciudadanía insurreccional de Balibar (2013). Es, siguiendo a ambos, el triunfo o el surgimiento dominador del individuo. Y es que, dice Bauman:

La otra cara de la individualización parece ser la corrosión y lenta desintegración del concepto de ciudadanía. (...) Si el individuo es el enemigo número uno del ciudadano, y si la individualización pone en aprietos la idea de ciudadanía y la política basada en ese principio, es porque las preocupaciones de los individuos en tanto tales colman hasta el borde el espacio público cuando éstos aducen ser los únicos ocupantes legítimos y expulsan a los codazos del discurso público a todos los demás. Lo "público" se encuentra colonizado por "lo privado". (Bauman, 2010, p. 42)

En la experiencia chilena, algunos procesos de creación y desarrollo de radios comunitarias muestran que son -en la práctica- experiencias liberadoras. Dos ejemplos nos ayudan a ilustrar esta situación. ${ }^{1}$

1 Ambos ejemplos corresponden a acciones de la iglesia católica chilena. Nos ha parecido pertinente citarlas ya que muestran una arista importante: a pesar de estar destinadas a la evangelización religiosa, su base está en la comunidad y en la participación. 
Con características únicas, se puede observar la protagonizada por el sacerdote de la Congregación Don Guarello, Antonio Ronchi (1930-1997), en la zona austral de Chile, quien entre las décadas del 60 y los 90 prácticamente "sembró" emisoras comunitarias en la Patagonia. Fueron 45 emisoras instaladas con y para la comunidad, algunas de las cuales sobreviven y conforman la red MADIPRO (Madre de la Divina Providencia), del Vicariato Apostólico de Aysén.

Cisternas cita al colaborador de Padre Ronchi, Narciso Nahuelquín, de Melinka, en el archipiélago de Las Guaitecas, en la región de Aysén:

\footnotetext{
Él decía que quería unir a la gente, porque algunos capaz que no le tengan mucho entusiasmo al rezo, rezar en la radio o todo el día pasar rezando en la radio, la gente no te va a pescar, pero si tú le tocas charrasqueados, los viejos igual me van a escuchar, decía él (...) y socializar más con la gente, eso era lo que pensaba como misionero, ese era su punto clave (Cisternas, 2014, p. 53)
}

En los 80 destacó otro proceso en el sur, esta vez en Chiloé, con Radio Estrella del Mar, y la creación de cabinas radiofónicas, a cargo de comunicadores populares. El obispo Juan Luis Ysern, de la Diócesis de la isla, al definir el proyecto de Radio Estrella del Mar y de la Fundación Estrella del Mar de Chiloé, y sus cabinas radiofónicas desperdigadas por islas, fiordos y poblados de la isla, para que la comunidad se intercomunicara, solía decir que a través de estos procesos a veces no se abrían puertas, pero si se abrían ventanas. ${ }^{2}$

El ejemplo de Ronchi y la metáfora de Ysern ilustran que la instalación de estos medios son procesos ejemplificadores y amplificadores del ejercicio de los derechos de las personas, pero que en definitiva son éstas, en comunidad, las que llevan adelante los procesos de liberación y cambio.

\section{Descripción de las radios comunitarias en $\mathrm{Chile}^{3}$}

La primera etapa de esta investigación recogió información de radios comunitarias -con y sin concesión legal- con el fin de obtener los datos que sirven de base para una posterior muestra de entrevistas semiestructuradas. El catastro obtuvo información de 44 emisoras

\footnotetext{
2 Intervención del obispo de Ancud, monseñor Juan Luis Ysern, al recibir el Premio Luis Oyarzún en la Universidad Austral de Chile, Valdivia, el 8 de septiembre de 2005. Archivo personal del autor.

3 La información completa del Catastro está en Ramírez, J.D. (2014) La radio comunitaria en Chile: perfil de las emisoras ciudadanas en 8 regiones del país. Ponencia XII Congreso ALAIC, agosto 2014, Lima. ISSN 2179-7617. Disponible en http:// congreso.pucp.edu.pe/alaic2014/wp-content/uploads/2013/09/GT8-Juan-Domingo-Ram\%C3\%ADrez.pdf
} 
de 8 regiones del Norte Grande, Centro, Centro Sur y Sur de Chile, incluyendo dos regiones del Norte Grande, que inicialmente no estaban contempladas en el estudio. Fue realizado en el segundo semestre de 2013, período en que según cifras de la Subsecretaría de Telecomunicaciones (SUBTEL) ${ }^{4}$ había 269 radios comunitarias concesionadas, por lo que las regiones indicadas corresponden al 91\% del territorio en donde están las radios.

Las cifras de la SUBTEL indican que entre 2010 y 2014 el número de radios comunitarias concesionadas se mantiene estable, por sobre las 300, salvo el 2013, debido al proceso de migración que estableció la ley de Servicios Comunitarios y Ciudadanos de Radiodifusión de 2010.

A continuación se muestran algunos gráficos atingentes al presente artículo.

En la Figura 1 se observa una dispersión del modo en que las radios se vinculan con las audiencias, que se da principalmente con Agrupaciones Culturales y Juntas de Vecinos, así como con las iglesias católicas y evangélicas. Respecto al cómo se vinculan (Figura 2) declaran hacerlo a través de "Programas hechos por la comunidad" y "salen en la radio dando sus opiniones". Llama la atención el hecho de que no se declara una participación de la comunidad en la gestión de la emisora, lo que podría indicar un problema en la definición y en la operatividad de los principios comunitarios, unido a que se valoriza el "dar la voz", pero no se observa una mayor participación en su gestión.

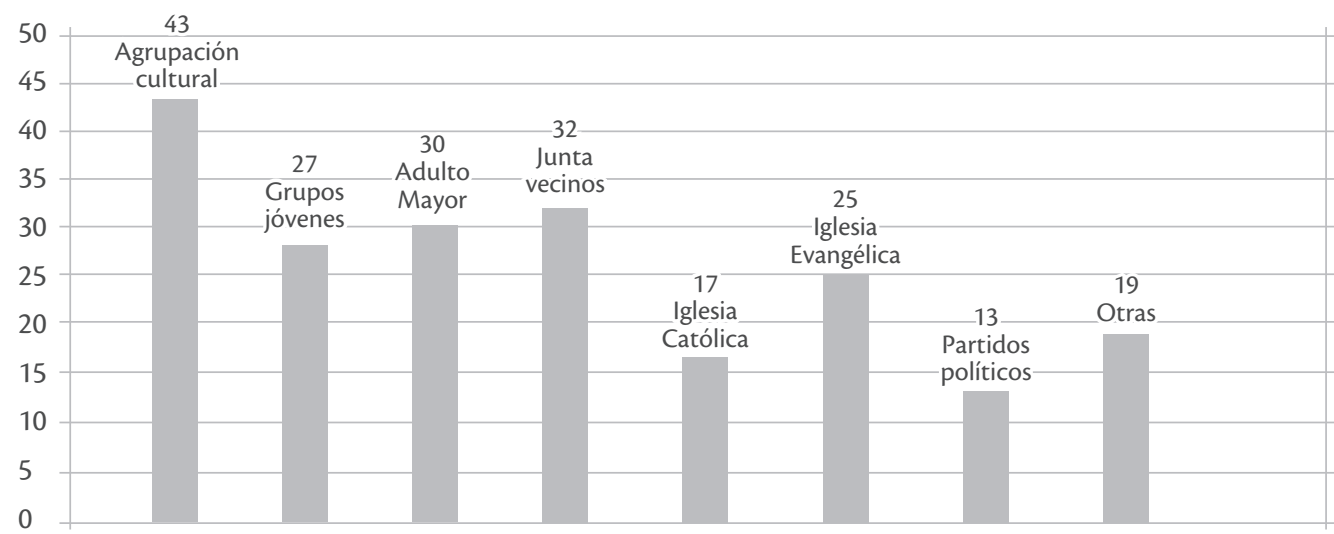

Figura 1: ¿Con qué organizaciones se vincula habitualmente? (Frecuencia de respuestas / $n=44$ ).

4 La Subsecretaría de Telecomunicaciones (SUBTEL) del Ministerio de Transportes y Telecomunicaciones de Chile es el organismo técnico encargado de las concesiones del espectro radioeléctrico chileno. 


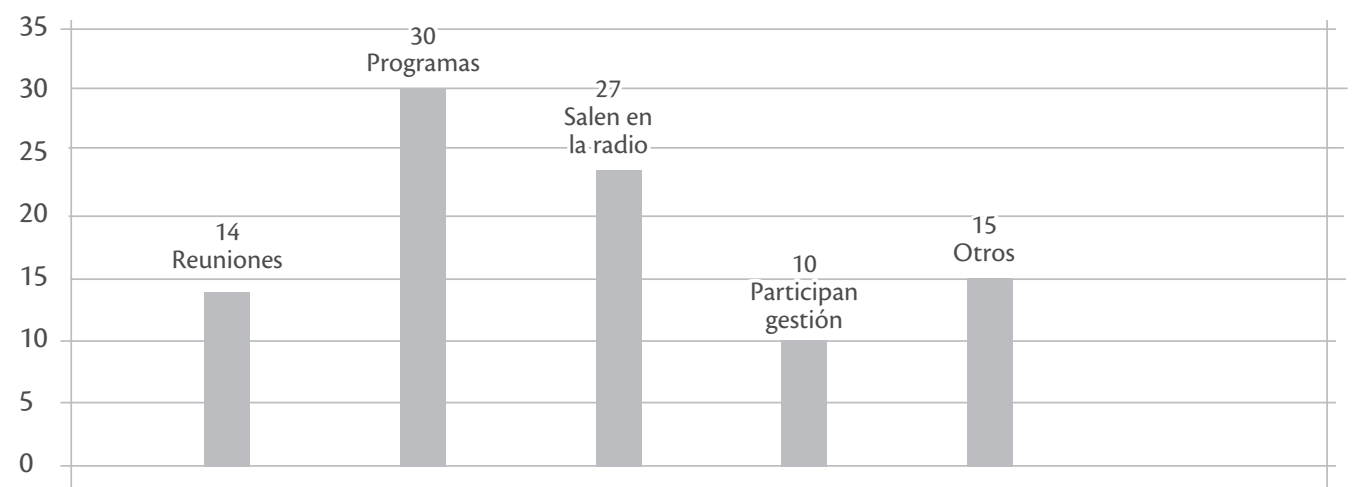

Figura 2: ¿Cómo se vincula con su comunidad? (Frecuencia de respuestas / $n=44$ )

Es en el uso de las tecnologías 2.0 se observa una debilidad, que puede explicarse, por un lado, por los bajos niveles de alfabetización digital en Chile, difícil acceso a conexiones de calidad y con la facilidad que ofrecen aplicaciones como Facebook y Twitter. Como se observa en la dispersión de la Figura 3, los soportes multimediales son precisamente Facebook y Twitter, así como la aplicación Wordpress, refrendado por las combinaciones de aplicaciones que muestra el Figura 4.

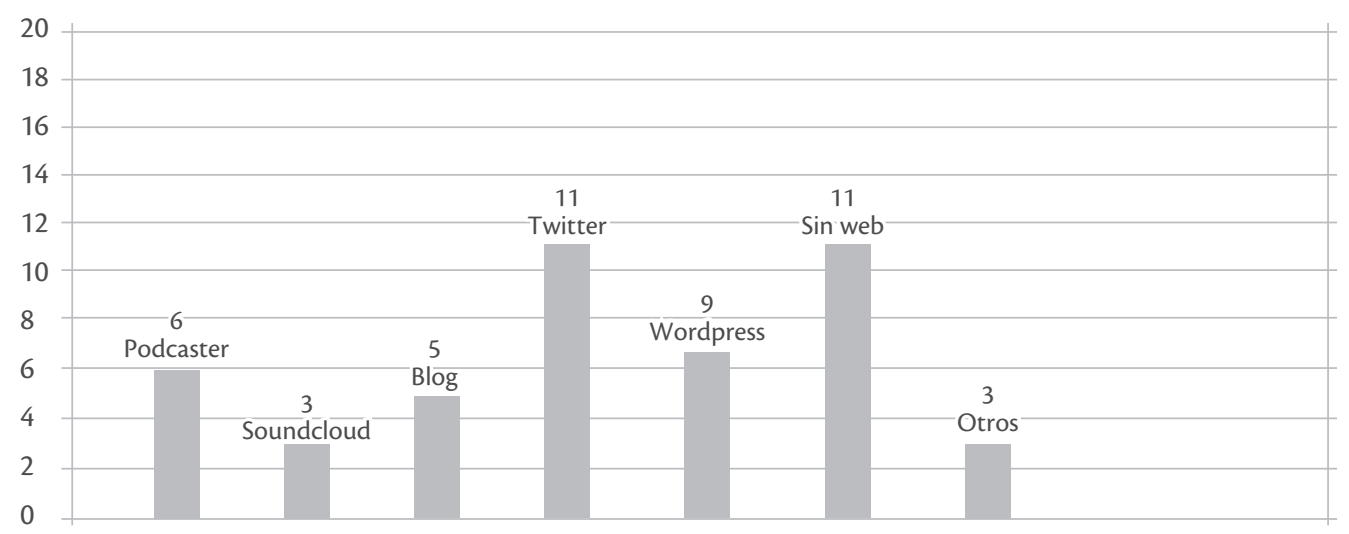

Figura 3: Aplicaciones usadas en la página web (dispersión)

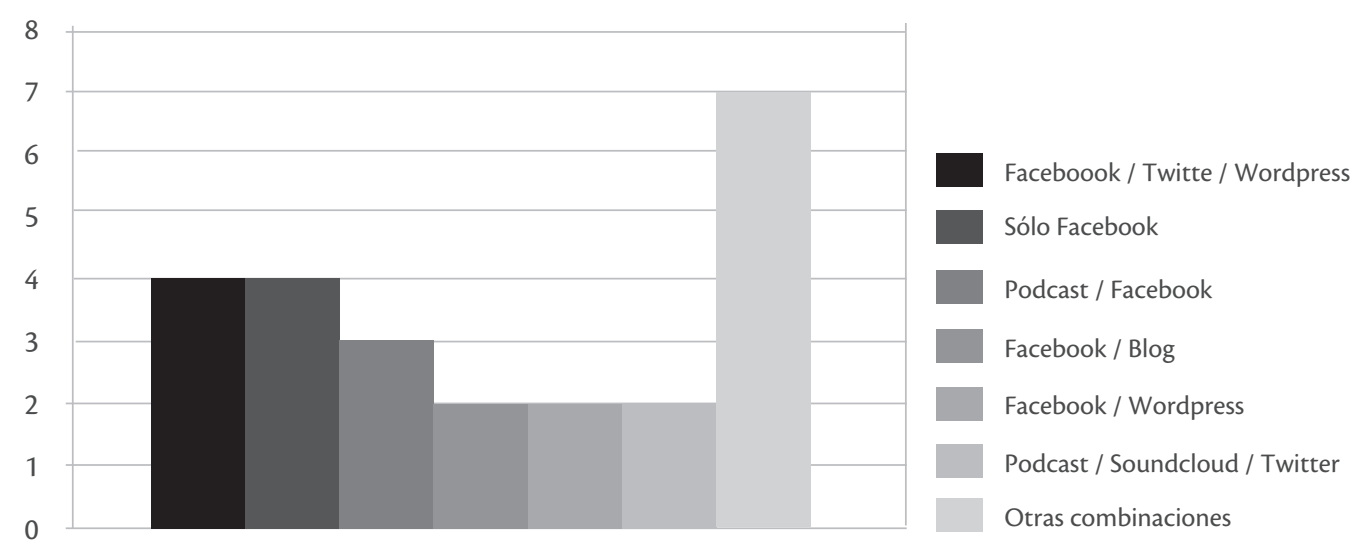

Figura 4: Combinaciones en uso de tecnologías web $2.0(\%$ - n=24) 
En el plano de la asociatividad (Figura 5) la mayoría pertenece a una o más organizaciones nacionales y/o internacionales (Anarcich y Amarc Chile), un 23\% no lo hace, ya sea porque las desconocen o no están interesadas, y sólo un $3 \%$ dice que no le interesa por desconfianza.

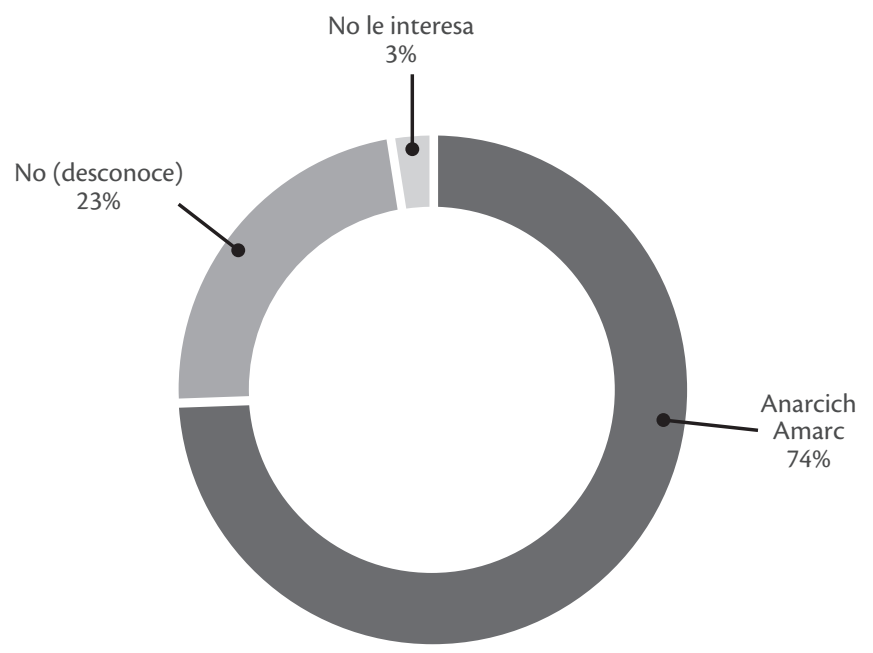

Figura 5: ¿Pertenece la radio a alguna organización?

\section{Método de análisis}

En esteartículo se presentan los resultados preliminares de las entrevistas semiestructuradas a directivos de emisoras comunitarias, en este caso, a una muestra de 4 entrevistas en la zona Centro Sur y Sur de Chile, distribuidas como se observa en la Tabla 1. Dicho análisis se efectuó con el software Atlas.ti que se basa en la Teoría Fundamentada (Grounded Theory) desarrollada por Glaser y Strauss (1967) y Strauss y Corbin (2002).

Tabla 1: Distribución de la muestra de análisis

\begin{tabular}{|l|c|}
\hline \multicolumn{1}{|c|}{ Zona geográfica } & Muestra de análisis \\
\hline Centro Sur: VII región del Maule - VIII región del Bio Bio & 2 \\
\hline Sur: IX región de la Araucanía - XIV región de Los Ríos & 2 \\
\hline
\end{tabular}

Fuente: Elaboración propia.

En metodología cualitativa, la Teoría Fundamentada es más bien una perspectiva de análisis más que un método final, es decir, permite la revisión exhaustiva de los datos, 
generando de los mismos relaciones, unidades de análisis $y$, a partir de esto, permite construir teoría. De los datos, la interpretación constante del investigador permite una construcción constante, cuya finalización está solamente en la saturación.

El programa Atlas.ti se transforma en una herramienta -no es el método en sí- facilitando la exploración, la codificación y la sistematización. El análisis se realiza a partir de la construcción de una Unidad Hermenéutica que consta de:

a) Documentos primarios: en este caso, las entrevistas.

b) Citas: que son los fragmentos elegidos por el investigador, tras una lectura constante y sucesiva de los Documentos Primarios.

c) Códigos: son las unidades básicas de los análisis, determinados por el investigador en función de la información y relaciones que se busca establecer. Los códigos pueden estar en constante movimiento ya que lecturas sucesivas pueden agruparlos, eliminarlos o crear nuevos. Se relacionan con la estructura de recolección de datos que se efectuó en la entrevista, aunque pueden surgir nuevos de la lectura constante.

d) Familias: unidad que permite agrupar los Documentos Primarios, Códigos y anotaciones que van surgiendo en el análisis.

e) Redes: aplicación visual que permite representar de manera gráfica las relaciones que se han establecido entre los componentes de la Unidad Hermenéutica.

El programa permite, además, la elaboración de Memos y Anotaciones, que son las reflexiones prácticas y teóricas que van surgiendo durante el proceso y que más tarde permitirán construir de manera menos compleja la teoría.

Tanto los métodos cualitativos como la utilización de software de análisis de datos aún tienen cuestionamientos en las ciencias sociales. Cabe hacer presente que Atlas.ti es sólo una herramienta que representa de igual modo "el papel, el lápiz y los marcadores" utilizados anteriormente en el análisis de datos cualitativos, en este caso del discurso de los entrevistados. Es el investigador quien estructura, organiza unidades, conceptos, procesos, buscando las redes que están tras los datos. Es simplemente un facilitador que remplaza los papeles recortados y categorizados de los análisis que anteriormente se realizaban de manera manual. 


\section{Resultados}

Para la realización del análisis se definió lo siguiente Unidad Hermenéutica:

a) Documentos Primarios (DP): cuatro entrevistas denominadas Centro Sur 1, Centro Sur 2, Sur 3 y Sur 4.

b) Códigos: se utilizaron 9 códigos para el análisis de los DP.

c) Familias: se agruparon los Códigos en 4 familias.

Dada la confidencialidad de las entrevistas y el hecho de que algunas radios no poseen concesión, transmiten con mayor potencia, tienen altura de antenas mayor que la permitida, se ha codificado la identificación de las emisoras. Asimismo, en los textos de las conversaciones se han eliminado referencias al nombre de la radio y referencias al lugar donde se ubican.

Tabla 2: Unidad Hermenéutica Atlas.ti

\begin{tabular}{|c|c|c|}
\hline $\begin{array}{l}\text { DOCUMENTOS } \\
\text { PRIMARIOS (DP) }\end{array}$ & FAMILIAS & CODIGOS \\
\hline \multirow{4}{*}{$\begin{array}{l}\text { P1: Centro Sur } 1 \\
\text { P2: Centro sur } 2 \\
\text { P3: Sur } 3 \\
\text { P3: Sur } 4\end{array}$} & Asociatividad & $\begin{array}{l}\text { Dificultades Asociación: porqué cuesta asociarse. } \\
\text { Participación asociaciones: porqué participan. }\end{array}$ \\
\hline & Comunidad & $\begin{array}{l}\text { Necesidades: lo que necesita la comunidad de la radio. } \\
\text { Participación: modo de participación en la radio. } \\
\text { Programas comunitarios: papel de la comunidad en los } \\
\text { programas. }\end{array}$ \\
\hline & Legislación & $\begin{array}{l}\text { Leyes: cómo ven las leyes radiales. } \\
\text { Publicidad: cómo mejora/dificulta las menciones pu- } \\
\text { blicitarias. }\end{array}$ \\
\hline & $\begin{array}{l}\text { Redes } \\
\text { Sociales }\end{array}$ & $\begin{array}{l}\text { Uso de Redes sociales: potencialidad/dificultad de uso. } \\
\text { Crecimiento Redes sociales: proyección de las redes. }\end{array}$ \\
\hline
\end{tabular}

Fuente: Elaboración propia.

A continuación se presentan las Redes resultantes del análisis sobre la base, como se señaló, de la Teoría Fundamentada con uso de software Atlas.ti. En dicho análisis se presentan también una selección de las intervenciones de las personas entrevistadas relacionadas con el tema revisado. 


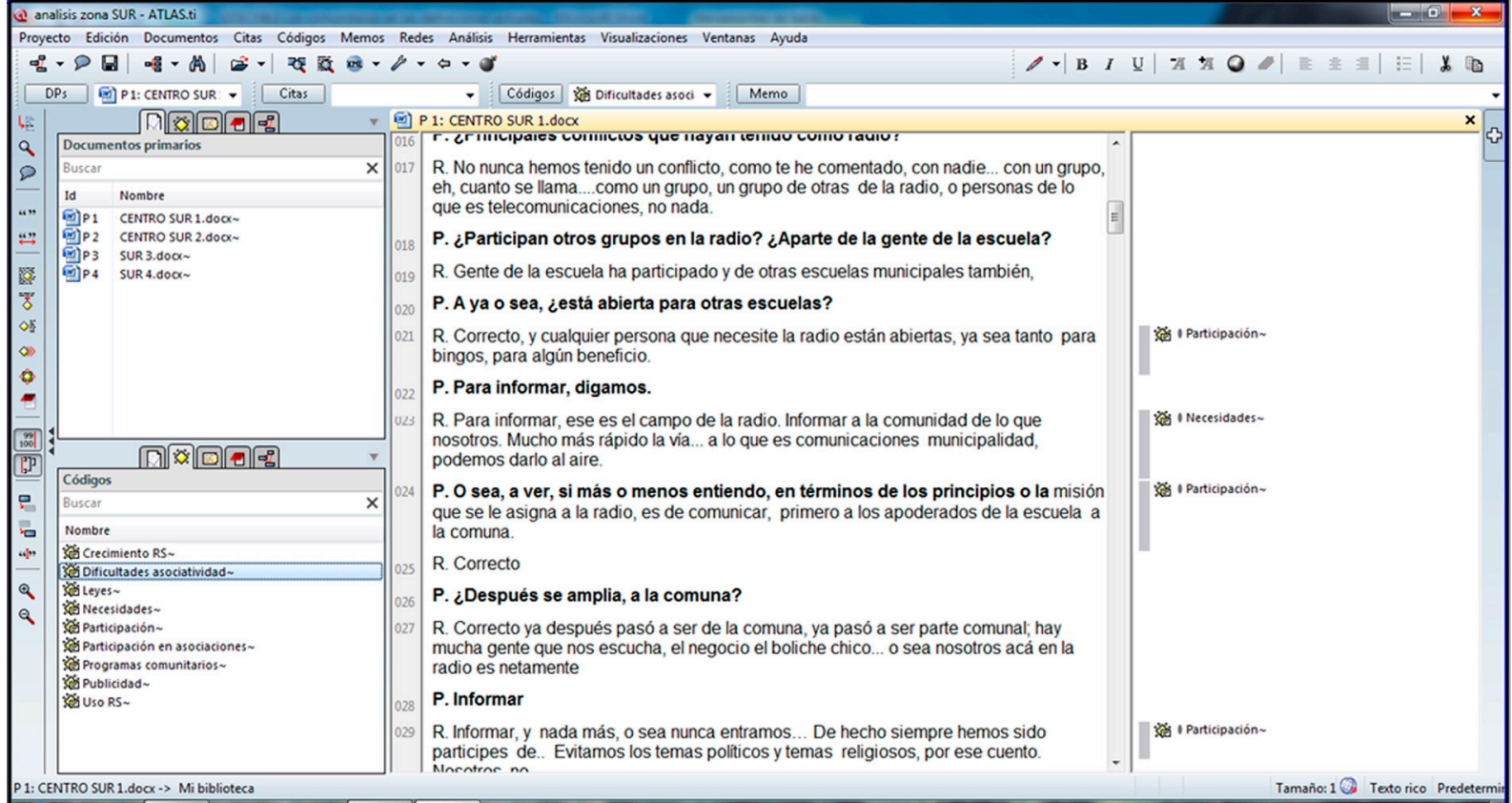

Figura 6: Captura pantalla análisis Atlas.ti

La Figura 6 muestra el estado inicial del análisis con Atlas.ti. El cuadro superior izquierdo muestra los DP; el inferior, los Códigos; al centro el DPanalizado, en este caso, el correspondiente a Centro Sur 1; y a la derecha, la asignación de Códigos que se realiza a lo largo de todo el DP seleccionado.

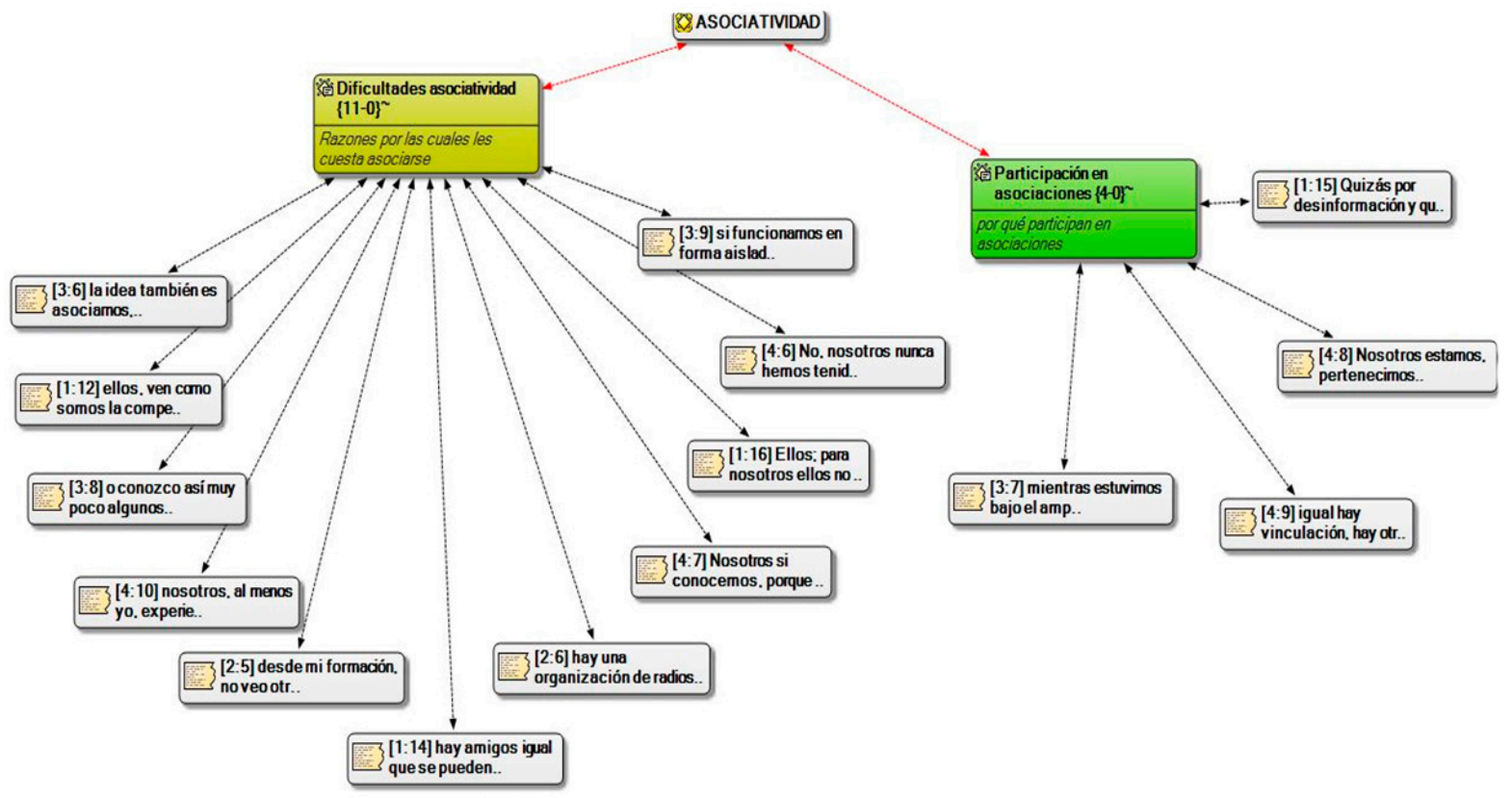

Figura 7: Red de Familia Asociatividad. 
La Figura 7 presenta la Familia Asociatividad que fue vinculada con dos códigos: Dificultades de asociatividad y Participación en Asociaciones. La red da cuenta que hay pocas emisoras que directa y activamente participan en organizaciones que las reúnen (código de la derecha, en verde), mientras las relaciones que más se observan están en el código Dificultades de Asociatividad (código izquierdo, en amarillo). Ello porque si bien estiman que la participación en organizaciones es importante, no lo hacen por desconocimiento o porque hacen equivalente asociarse a competir con otros medios, a pesar de no llegar a los mismos públicos.

1:12 ellos, ven como somos la competencia. Ellos...para nosotros ellos no son una competencia, ellos ven otro campo de público, de radio escuchas, ellos son más populares.

4.10 nosotros, al menos yo, experiencias de radio yo de verdad nunca he estado en otra radio que no sea la de nosotros...

3:9 si funcionamos en forma aislada no creo que va a salir más fácil si nos asociáramos si compartiéramos experiencias, materiales, compartimos programación, etc.

4.7 Nosotros sí conocemos, porque antes sí participábamos en congresos que hacia la Anarcich, pero cuando nosotros levantamos la radio acá, en los momentos complicados, difíciles que vivimos, cuando llego el tema judicial en contra la radio, nosotros pedimos ayuda por todos lados y fue la Amarc la que llego acá...

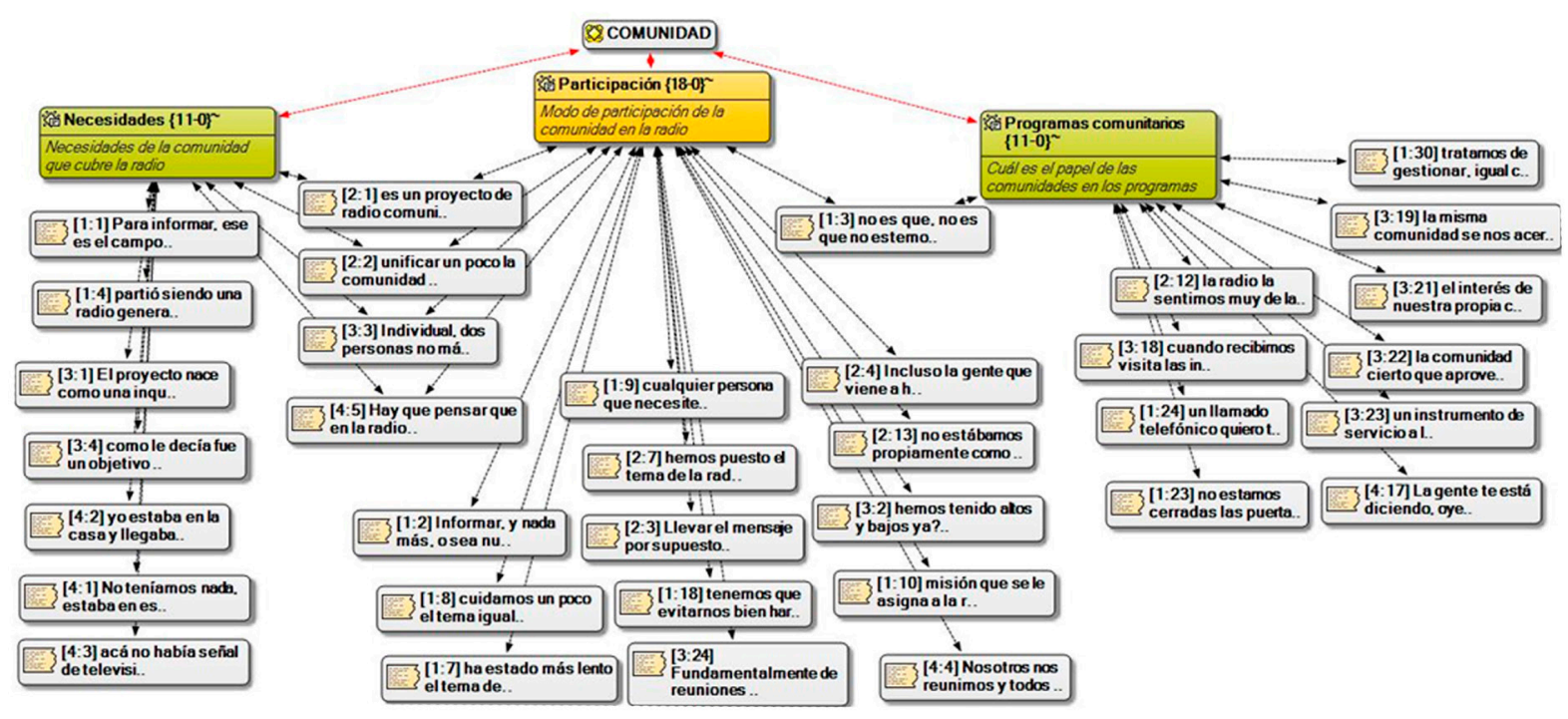

Figura 8: Red de Familia Comunidad 
La Figura 8 corresponde a la familia Comunidad que es el más complejo y se observará en detalle. Tal como se vislumbró en el catastro ya citado, si bien es amplio el discurso de la Participación y el nacimiento de las radios para cubrir Necesidades de la comunidad, la participación de las audiencias (entendidas también como comunidad objetivo) en la propuesta y elaboración de programas propios, así como en la gestión de la emisora está poco relacionada. Es decir, se puede observar en la red que están mucho más claras las Necesidades de la comunidad y la Participación de la misma en la emisora, pero se relacionan más que todo con los deseos que con una expresión visible en los programas radiales o la gestión de la emisora.

3:24 Fundamentalmente de reuniones y comunicación, lazos personales porque casi todos nos conocemos acá.

1:7 ha estado más lento el tema de participación de los alumnos por un tema de que, el curso que participaba, está entrando en otra etapa de su educación...

3:4 como le decía fue un objetivo pedagógico, pero hoy en día más que el objetivo pedagógico ya está basado en el servicio a la comunidad, en proyectarla a la comunidad y toda sus necesidades de comunicación,

4.2 yo estaba en la casa y llegaba acá una sola radio de la comuna, una radio comercial pero que tenía muy poco contenido de la comuna y no tenía nada de contenido de las comunidades indígenas sobre todo.

2:2 unificar un poco la comunidad y la organización de hecho le dimos harto, harto al tema, de la unidad, y mi propia experiencia, en la organización, me indicaba que faltaba ese mensaje, y que hizo mucho eco tal vez porque era un poco dado por él, lado cristiano...

3:21 el interés de nuestra propia comunidad de que ellos aprecien el esfuerzo que se ha hecho por tener esta concesión radial que la comunidad también la aproveche.

1:24 un llamado telefónico quiero tal tema, pero los fines de semana, o tardes noches, va grabado, va seleccionado, aleatoria, o recuerdo, ochenta, romántico, ya ese es el estilo

3:18 cuando recibimos visita las invitamos a que se dirijan a la comunidad en forma emergente. 


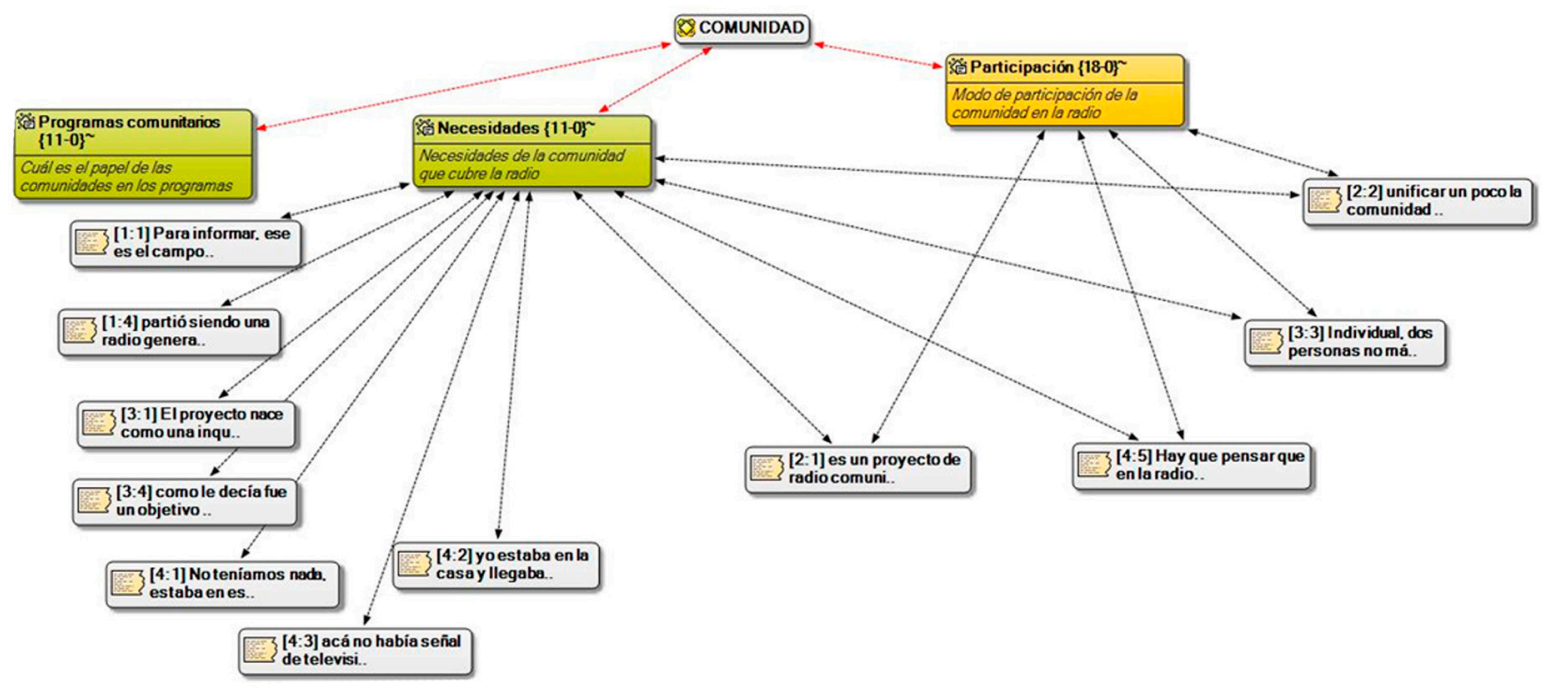

Figura 9: Red de Familia Comunidad, relaciones Participación y Necesidades

Esta red representada en la Figura 9 desagrega lo dicho anteriormente, vinculando el discurso de Participación y Necesidades, los cuales, mirado exclusivamente desde esta perspectiva, no desarrollan redes de relación con los Programas Comunitarios.

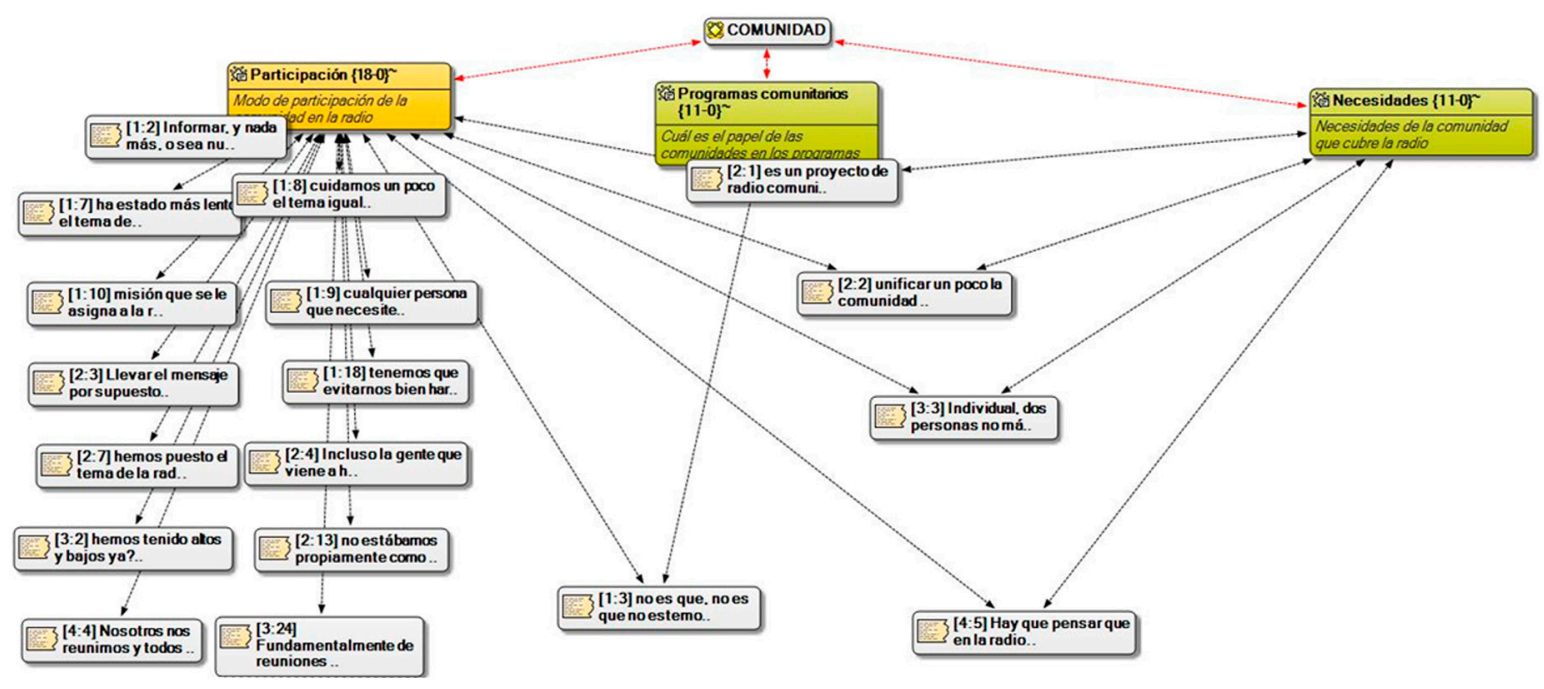

Figura 10: Red de Familia Comunidad, relaciones Programas Comunitarios

El mismo desarrollo anterior muestra la Figura 10 donde se mira el discurso desde la perspectiva de los Programa comunitarios, donde su descripción se relaciona en un bajo nivel con las Necesidades y la Participación. 

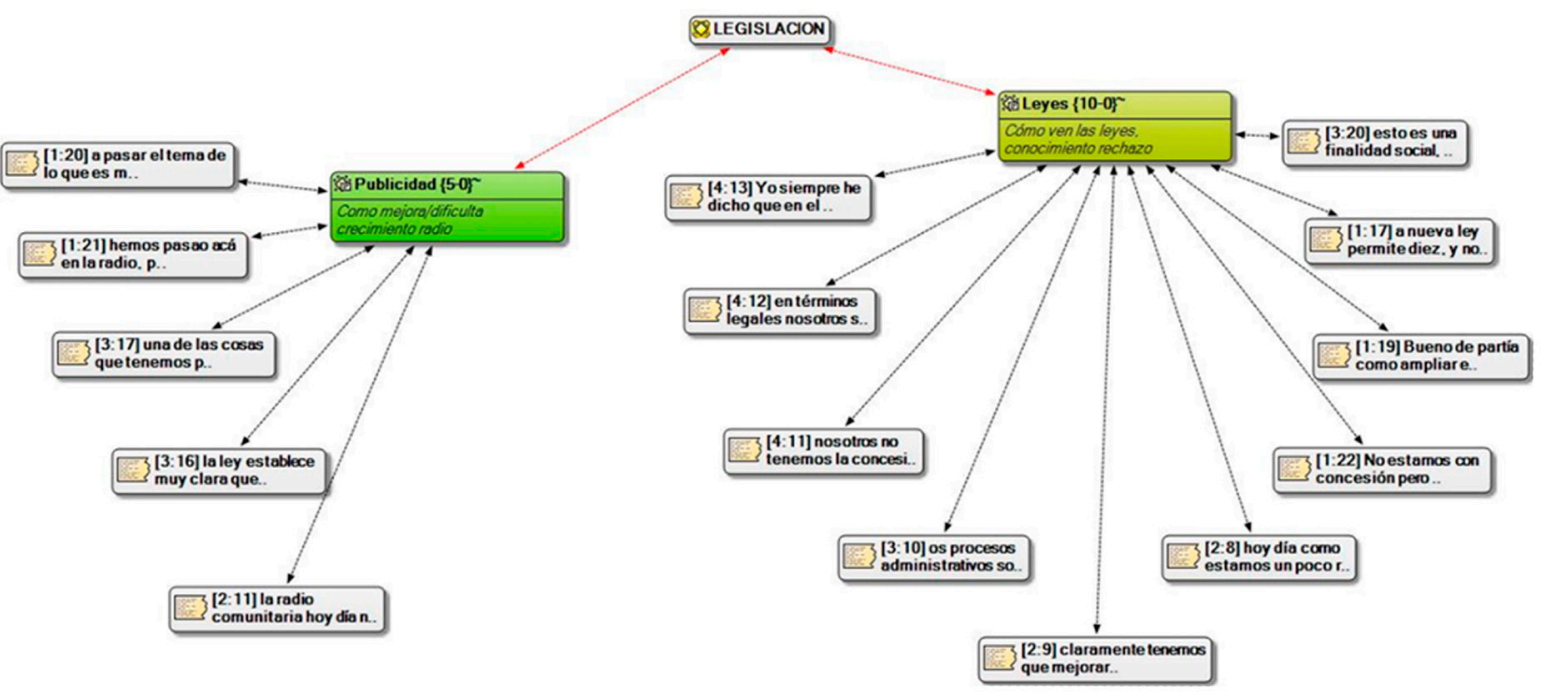

Figura 11: Red de Familia Legislación

La Figura 11 muestra las relaciones de red que se establecen desde la familia Legislación. Al rechazo mayoritario de la actual ley, se observa un interés en la posibilidad de realizar menciones publicitarias (que para facilitar la lectura se ha nombrado como el código Publicidad). Aunque se valora el cambio, se observa que no es un elemento que ayude a la sostenibilidad de las emisoras, sobre todo por las dificultades relacionadas con la iniciación de actividades que deben realizar, para el pago de los impuestos por estas transacciones menores.

4:13 Yo siempre he dicho que en el tema de las radios comunitarias está todo tan cuadradito, todo tan diseñado para que estas radios no funcionen...

1:22 No estamos con concesión pero por lo que leímos una vez hay una parte, que estamos bajo el alero educacional.

1:19 Bueno de partida como ampliar el tema de la potencia igual favorece en algo...Y también lo que lo favorece mucho, yo creo que nos favorece...

3:20 esto es una finalidad social, no tenemos fines de lucro, el fin es prestar un servicio a la comunidad así que no se cambie la legislación, que se fortalezca. 
1:20 a pasar el tema de lo que es menciones, ya está más abierto a eso, porque antes, claro, se podían hacer menciones, pero (....) disfrazándolas como colaborando.... ya ahora es una mención más, mas, abierta...

3:17 una de las cosas que tenemos pendiente es el inicio de actividad, por eso nosotros tenemos un rut para el servicio de impuestos internos, pero nos falta hacer la iniciación de actividades.

2:11 la radio comunitaria hoy día no puede hacer menciones, entonces como se financia para poder ampliarse.

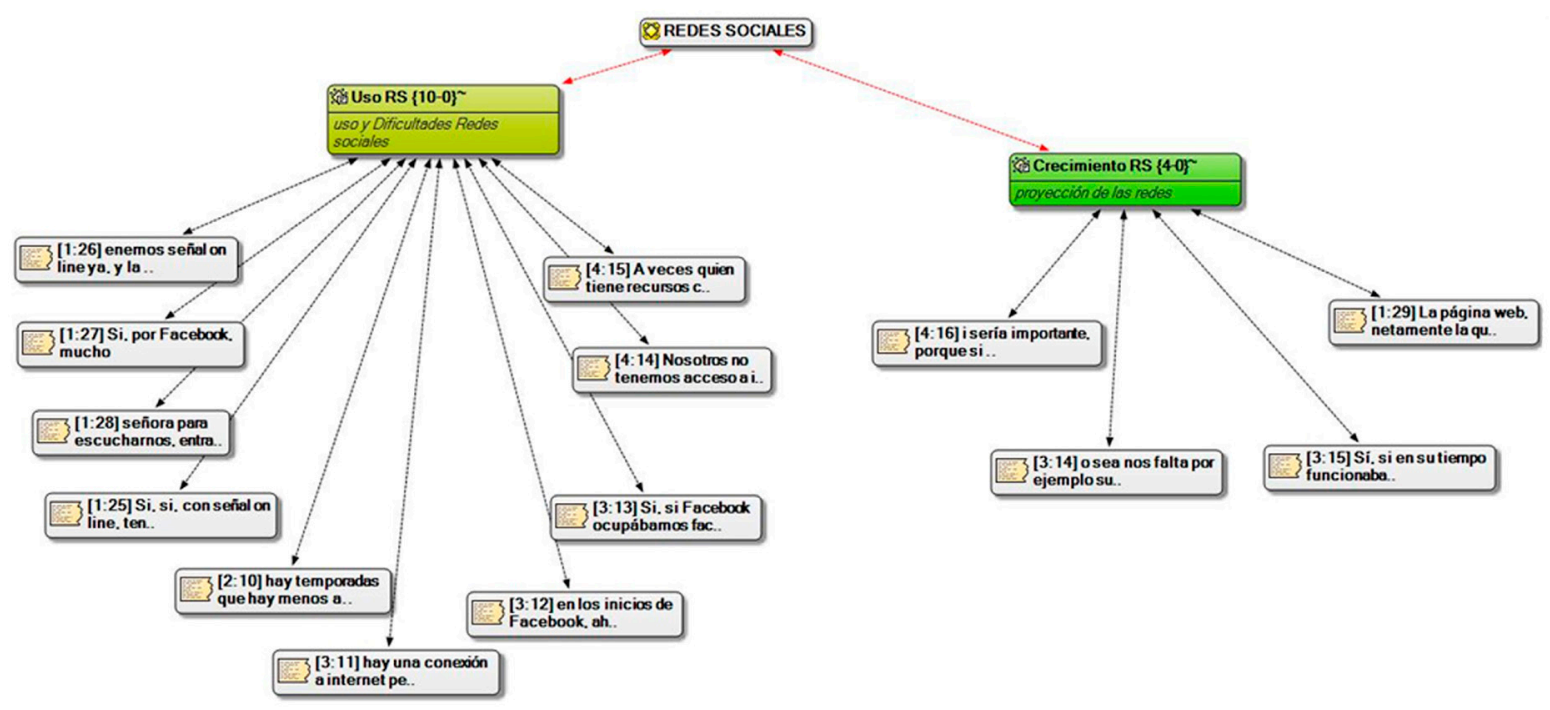

Figura 12: Red de Familia Redes Sociales

La Figura 12 corresponde a la red de la Familia Redes Sociales, indagando sobre el Uso y el Crecimiento de las mismas. Se observa que hay una relación estrecha en el conocimiento y uso de las mismas, pero en niveles usuarios con las redes Facebook y Twitter, así como en el uso de software gratuitos de transmisión y streaming. El relato habla de un nivel instrumental, ya que no hay una clara relación entre el Uso y el Crecimiento de las mismas, es decir, en cómo potenciarlas para ampliar la cobertura de la emisora en términos de audiencia/auditores y amplificación del mensaje de los grupos que operan las emisoras.

1:26 tenemos señal on line ya, y la web tampoco es una web muy... eh con mucho, eh.... muy difícil de acceder, ya porque nosotros muchas señoras trabajan o vecinas de acá que trabajan en (...) y nos llaman... 
3:11 hay una conexión a internet pero sirve para actualizar información, para noticias para que el operador cierto este bajando noticias todas esas cosas pero no tenemos esa posibilidad.

4:14/4:15 Nosotros no tenemos acceso a internet y lo que las personas que hacemos los programas buscamos los medios de como poder entregar información (...). A veces quien tiene recursos carga su modem y tiene conexión a internet.

3:14 o sea nos falta por ejemplo subirnos, tener una buena red de internet, una buena, y eso mismo no cierto nos puede permitir subir a la radio a la atmosfera, a la web no lo tenemos en este instante.

1:29 La página web, netamente la que tenemos ahora que es poca la información que podemos dar ya, hoy la tenemos ligada al tema de lo que es notica, ya...

\section{Conclusiones}

Al inicio se señaló que el movimiento social en torno a las radios comunitarias no debe observarse de manera estática, ya que su riqueza se encuentra en los procesos que se experimentan tanto dentro de los grupos que las operan, como en el entorno que se constituye en las audiencias. Por lo tanto, el título de este acápite debiera ser más bien "a modo de conclusiones", ya que se refiere a mirar en un momento el discurso comunitario, en voz de las personas que las hacen, e intentar entender y explicar sus contenidos.

La Teoría Fundamentada es una muy buena herramienta para estos casos, ya que está centrada en los datos obtenidos y en cómo, a partir de éstos, se va construyendo teoría, desde la visión del investigador; al ser los datos obtenidos en entrevistas, elementos "vivos" de un discurso, el investigador debe estar atento a obtener la mayor fidelidad de éstos en sus resultados. Consecuentemente, el software Atlas.ti permite buscar respuestas, llegando a un aparente final de análisis - poco posible con la vida de los mismos datos- al llegar a niveles de saturación.

El artículo muestra tres niveles: Comunitario/ciudadano, información catastral y análisis de entrevistas semiestructuradas. 
La irrupción del concepto de ciudadanía en la definición de las radios, si bien da cuenta de un fenómeno de ruptura de los límites geográficos para las emisoras, también debe observarse cuidadosamente, ya que por la misma diversidad de los medios comunitarios, es posible que esta definición no se adapte como generalidad a los distintos procesos de este movimiento. Lo comunitario, pequeño, barrial, sigue teniendo un impacto profundo tanto en los movimientos que gestan estas radios como en las audiencias. Lo que se propone, desde la discusión que se ha hecho, es estar atento a las especificidades de estos medios, optando por las definiciones operativas lo más amplias posibles.

La información catastral obtenida -y entregada en forma resumida en este artículo-, los datos que arrojan las entrevistas y su análisis desde la Teoría Fundamentada, permiten establecer una mirada global al estado del movimiento de radios comunitarias en Chile, aunque este autor prefiere hablar de "fotografía", para enfatizar el carácter del proceso.

¿Qué muestra esta fotografía? Tomando las familias que se determinaron para los Documentos Primarios, podemos observar:

En Asociatividad existe claridad en que juntos se puede trabajar mejor y la mayoría de las emisoras conocen las asociaciones que existen. Pertenecen mayoritariamente - y de manera paralela en algunos casos- a la Asociación nacional de radios comunitarias y ciudadanas de Chile (ANARCICH) y a la sección chilena de la Asociación Mundial de Radios Comunitarias (AMARC). Valoran el poder reunirse con otras emisoras, pero al menos surgen dos elementos a poner atención. Uno es un grado de desconfianza, ya que -en sus palabras- muchos radialistas se ven como competencia unos de otros, más que como integrantes de un movimiento social cohesionado; el otro, es el desconocimiento de cómo funcionan estas asociaciones, ya sea por la distancia o por la falta de conversación y vínculo entre las propias radios y entre éstas y las organizaciones.

En Legislación el conocimiento es alto, toda vez que lo técnico está regulado por la ley (potencia, altura de antena, ubicación de estudios). Obviamente, hay un rechazo al marco legal actual, aunque -relacionado con el punto anterior-no se articula con otras emisoras o movimientos. Incluso el hecho que la ley de 2010 permitiera "menciones comerciales" -no publicidad directa- es visto como algo favorable, pero limitado por el hecho de tener que realizar un trámite de "iniciación de actividades" ante el Servicio e Impuestos Internos y llevar contabilidad. 
En Redes Sociales se observa -como se había visto en los resultados del catastro- un uso intensivo de Facebook y Twitter, aunque intermitente entre la red de la radio y las cuentas de los encargados de cada programa transmitido. Lo mismo, al existir un buen número de radios que transmiten on line, hay una correcta operación de software de transmisión y de streaming. Sin embargo, al observar el árbol de red, no existe gran relación en este uso instrumental y la idea de crecimiento de éstas, como una forma de conectarse más con sus audiencias o crear nuevas.

Comunidad es la familia que puede calificarse como la más interesante en resultados $y$ es concordante con lo que se ha observado en otras investigaciones de este autor. $\mathrm{El}$ discurso sobre la importancia de responder a la comunidad y satisfacer sus necesidades es importante, bonito e impactante. De hecho, es coincidente en emisoras comunales, urbanas, rurales, juveniles, étnicas, universitarias; las menciones codificadas en Atlas.ti son las mayores, según puede observarse en las redes. Está claro que las emisoras se crean y se mantienen para la comunidad y su expresión. No obstante, hay un dato que es inquietante y que las redes permiten observar: la poca relación entre este discurso comunitario y la participación de las audiencias en la radio, no solamente haciendo programas o saliendo en la radio, sino en los procesos de gestión de las emisoras.

En resumen, se trata de radios que se forjan en/y para la Comunidad, siendo las personas que la integran el objeto y el sujeto del sentido último de su creación. Esta comunidad en torno a la radio necesariamente tiene vínculos de Asociatividad, tanto entre sí como con otras radios y organizaciones radialistas. Su expresión, si bien es libre, está ligada a la Legislación con que pueden operar y si en las décadas pasadas su expresión era a través del aire, en la actualidad usan internet y las Redes Sociales para desarrollarse y por su intermedio intentar captar nuevas audiencias.

Y es acá donde los espacios de las emisoras comunitarias, concebidas - desde distintas perspectivas y entre otros tópicos- como lugar de encuentro, de gestión comunitaria, de ejercicio de derechos, insurrección, etc., deben definirse y quizá volver o mantenerse dentro de la esfera de la metáfora López Vigil, referida a un espacio común, una plaza común, donde todas las voces tengan lugar. 
Referencias Abrutin, M. (2006). Reflexiones en torno a la ciudadanía europea: iqué significan las fronteras de Europa? Ponencia III Congreso de relaciones internacionales. Universidad Nacional de La Plata, Argentina. Disponible en http://sedici.unlp.edu.ar/handle/10915/36943

Araya, R. (2007) Ciudadanía cultural: al rescate del espacio público. En Consejo Nacional de la Cultura de Chile. Ciudadanía, Participación y cultura. Santiago, Chile: Lom editores.

Balibar É. (2013) Ciudadanía.Buenos Aires: Adriana Hidalgo Editora Colección Fundamentos.

Bauman, Z., (2010). La modernidad Líquida. 1ra edición. 12a reimpresión. Buenos Aires: Fondo de cultura económica.

Bauman, Z. (2013). La cultura en el mundo de la modernidad líquida. Buenos Aires, Argentina: Fondo de Cultura Económica.

Cabalín Quijada, C. (2010) Democracia y comunicación: un lugar para los medios comunitarios. Revista Estudios F@ro no 11, 2010 Facultad de Ciencias Sociales, Universidad de Playa Ancha. Chile. Revista electrónica semestral http://web.upla.cl/revistafaro/n11/art01.htm

Cisternas, S. (2014) El sacerdote Ronchi y la formación de radios comunitarias en la región de Aysén. Tesis de grado Licenciatura Comunicación Social, Universidad Austral de Chile.

Chaparro, M. (2002) Sorprendiendo al futuro. Comunicación para el desarrollo e información Audiovisual. Serie Comunicación y Democracia. Ed. Los libros de la Frontera. Barcelona.

Glaser, B. y Strauss, A. (1967). The discovery of grounded theory. Chicago: Aldine.

López Vigil (2005) Manual Urgente para Radialistas Apasionados. Lima. Edición digital de código abierto. http://radioteca.net/media/uploads/manuales/2013_10/ManualUrgenteRadialistas.pdf

\section{Consultado marzo 2013.}

Martín Barbero, J. Tecnicidades, identidades, alteridades: desubicaciones y opacidades de la comunicación en el nuevo siglo. En De Moraes, D. (Ed). 2007. Sociedad Mediatizada. Gedisa, Barcelona.

Ramírez C., Juan Domingo (2010) Radios Comunitarias en Chile: las paradojas de su propiedad. Revista Austral de Ciencias Sociales, 2010, no.19, pp.63-74.

Ramírez C., Juan Domingo (2014) La radio comunitaria en Chile: perfil de las emisoras en 8 regiones del país. Anales XII Congreso latinoamericano de investigadores de la Comunicación. ISSN2179-7617. Lima. Disponible en http://congreso.pucp.edu.pe/alaic2014/wp-content/uploads/2013/09/GT8-Juan-DomingoRam\%C3\%ADrez.pdf

Ramírez., J. D. (2014): ¿Radios comunitarias, ciudadanas, populares? Las paradojas (antinomias) de una definición. Ponencia I Congreso Nacional Investigadores de la Comunicación (INCOM). Pucón, Chile. Octubre.

Rovira-Sancho, G, (2013) Activismo mediático y criminalización de la protesta: medios y movimientos sociales en México. Convergencia. Revista de Ciencias Sociales, vol. 20 n 61 enero abril 2013 pp. 35 - 60 UNAM.

Pasquali, A. 2014) Hay que cerrar las escuelas de comunicación y refundarlas. En www.prodavinci.com. Entrevista a Pasquali de Linares, A. http://prodavinci.com/2014/07/14/actualidad/antonio-pasquali-hayque-cerrar-las-escuelas-de-comunicacion-y-refundarlas-una-entrevista-de-albinson-linares/(Julio de 2014)

Strauss, A. y Corbin, J. (2002). Bases de la investigación cualitativa. Técnicas y procedimientos para desarrollar la Teoría Fundamentada. Medellín. Universidad de Antioquia. 
Van Oeyen, V. (2003) Los desafíos de la radio popular y comunitaria en américa latina. Ponencia IV Conferencia Internacional de Comunicación Social: Perspectivas de la Comunicación para el Cambio Social y el Tercer Encuentro Our Media/Nuestros Medios. Universidad del Norte. Barranquilla, 19-21 de mayo de 2003 http://buenosdiasamerica.blogia.com/2004/120103-los-desafios-de-la-radio-popular-y-comunitariaen-america-latina.php

Ysern, J.L. (2005) Discurso el premio Luis Oyarzun, otorgado por la Universidad Austral de Chile. Septiembre, 2005.

Yáñez, Collet, Weibel. (2011) Informe final Comprensión del fenómeno interrelacional entre productores de la radiodifusión comunitaria y audiencias comunales. Conicyt, Chile. Consultado en diciembre 2013 http:// www.conicyt.cl/informacioncientifica/files/2010/09/articles-37187_arcis_infFinalCompleto.pdf 